OPEN ACCESS

Edited by:

Robert E. Gross,

Emory University, United States

Reviewed by:

Hiroki Toda,

Fukui Red Cross Hospital, Japan Vibhor Krishna

The Ohio State University, United States

Konstantin Slavin,

University of Illinois at Chicago,

United States

*Correspondence:

Marc N. Gallay

marc.gallay@gmail.com

Specialty section:

This article was submitted to Neurosurgery,

a section of the journal

Frontiers in Surgery

Received: 04 January 2019 Accepted: 02 May 2019

Published: 17 May 2019

Citation:

Gallay MN, Moser D, Federau C and Jeanmonod D (2019) Radiological and Thermal Dose Correlations in Pallidothalamic Tractotomy With MRgFUS. Front. Surg. 6:28 doi: 10.3389/fsurg.2019.00028

\section{Radiological and Thermal Dose Correlations in Pallidothalamic Tractotomy With MRgFUS}

\author{
Marc N. Gallay ${ }^{1 *}$, David Moser ${ }^{1}$, Christian Federau ${ }^{2,3}$ and Daniel Jeanmonod ${ }^{1}$ \\ ${ }^{1}$ SoniModul, Center for Ultrasound Functional Neurosurgery, Solothurn, Switzerland, ${ }^{2}$ ETH Zurich, Institute for Biomedical \\ Engineering, University Zurich, Zurich, Switzerland, ${ }^{3}$ Department of Radiology, University Hospital Basel, Basel, Switzerland
}

Background: MR-guided focused ultrasound (MRgFUS) offers the possibility of safe and accurate lesioning inside the brain. Until now, most MRgFUS thermal applications have been based on temperature or energy protocols. Experimental studies support however an approach centered on thermal dose control.

Objective: To show the technical feasibility and lesion size predictability of a thermal dose approach during MRgFUS pallidothalamic tractotomy (PTT) against chronic therapy-resistant Parkinson's disease (PD).

Methods: MR and thermal dose data were analyzed in 31 MRgFUS interventions between January and December 2017 in patients suffering from chronic therapy-resistant Parkinson's disease (PD) using a standardized PTT target covered by 5 to 7 target lesion sub-units.

Results: Good correlations were found between (1) the mean axial T2 lesion diameter intraoperatively and the mean 240 cumulative equivalent min at $43^{\circ} \mathrm{C}(240 \mathrm{CEM})$ thermal dose diameter $(r=0.52)$, (2) the mean axial T2 diameter $48 \mathrm{~h}$ post-treatment and the mean 18 CEM thermal dose diameter $(r=0.62)$, and (3) the mean axial T2 diameter intraoperatively and $48 \mathrm{~h}$ post-treatment $(r=0.62)$.

Conclusion: Our current approach using a thermal dose steering for multiple target lesion sub-units could be reproduced in 31 interventions with a good lesion size predictability.

Keywords: MRgFUS (magnetic resonance-guided focused ultrasound surgery), Parkinson's disease, functional neurosurgery, pallidothalamic tractotomy, incisionless, thermal dose, imaging, radiology

\section{INTRODUCTION}

Our previous experience of lesioning the pallidothalamic tract on its way to the thalamus in PD (1) has led us, after analysis of recurrences or partial symptom control, to develop a targeting protocol aiming at improved spatial lesion coverage of the target. Our current approach, served by a histological reappraisal of the pallidothalamic tract, adopts the strategy of applying a set of small thermolesions with shortest possible sonication durations, under thermal dose control and moving the focal point of the MRgFUS system onto preplanned coordinates (2). This is a study of thermal dose and imaging correlations aiming to show the technical feasibility and lesion size predictability applying a thermal dose approach, and to compare it with currently used approaches 
primarily based on temperature or energy protocols. The thermal dose approach is supported by experimental data on focused ultrasound based thermal tissue lesioning (3-5), which predict a $100 \%$ lesion probability at the value of 240 CEM.

\section{METHODS}

Our current atlas-based spatial coverage technique of MRgFUS PTT described in a companion paper (2) was applied in 31 interventions in 29 chronic therapy-resistant Parkinson's patients. Mean age at treatment was $67 \pm 11$ years. The targeting accuracy of our MRgFUS intervention experience has been published between 2012 and 2018 (1, 6-9). Clinical results will be presented elsewhere.

Focused ultrasound procedure were performed using the ExAblate Neuro device (InSightec, Haifa, Israel) in a 3-Tesla MR imaging system (GE Discovery 750, GE Healthcare, Milwaukee, WI, USA).

We applied a standardized PTT target lesioning by moving the focal point of the focused ultrasound system onto 5 to 7 preplanned positions. Each sonication had the shortest possible duration and the corresponding power in order to provide a thermal dose of 240 CEM at each focal point. This represents a conservative value corresponding to a $100 \%$ probability of lesion (3-5) in a volume of $1.5 \times 1.5 \times 3.0 \mathrm{~mm}$ corresponding to the focal region as given by the manufacturer. According to McDannold $(3,4)$, an 18 CEM thermal dose represents a 50\% probability for thermal damage. The ExAblate software provides integration and graphic 2D-representation of the applied thermal doses of 240 and 18 CEM for each performed sonication. Diameters of thermal dose surfaces were measured on axial MR planes directly on the ExAblate software. No volumetric thermal dose assessment was performed.

To obtain 240 CEM in each target sub-unit, some sonications had to be repeated. Because it is not established yet in which way the thermal dose of repetitive sonications on the same spot may be cumulated, the goal was always to reach a thermal dose of 240 CEM in one sonication.

Intraoperative MR T2 examinations were performed at the end of the intervention as early as possible after the last sonication, with the body coil and with the patient in treatment position. Postoperative MR examinations with the 32-channel head coil were performed 2 days after the intervention, except in one patient, who received it 1 day after. Diameters of thermal lesions were measured on axial T2 MR planes. No volumetric lesion assessment was performed. The thermal lesions, consisting of zones I and II according to Martin et al. (10) were clearly distinguishable from perilesional edema.

Three additional cases were treated before this period and serve to demonstrate the necessity and applicability of the strategy presented here.

Abbreviations: ICL, intercommissural line; MTT, mammillothalamic tract; IC, internal capsule; CEM, cumulative equivalent min at $43^{\circ} \mathrm{C}$; MCL, midcommissural line; MRgFUS, MR-guided high intensity focused ultrasound; PTT, pallidothalamic tractotomy; PD, Parkinson's disease.
Corticosteroids were routinely applied in all patients treated with this protocol, intravenously within $1 \mathrm{~h}$ following last sonication (usually $40 \mathrm{mg}$ Solumedrol) and after $12 \mathrm{~h}$. Dexamethasone $(3 \times 2 \mathrm{mg}$ per day) was given p.o. the following 3-4 days.

Correlations were assessed with Pearson's coefficient with agreement considered good for $0.4<\mathrm{r} \leq 0.75$, and excellent for $0.75<\mathrm{r}$.

All patients treated with this protocol signed an informed consent form after having been fully informed about the treatment, its results and risks. No ethical approval was sought because MRgFUS PTT is approved by the Swiss Health State Department, is the subject of a state registry and is covered by swiss social insurances.

\section{RESULTS}

Figure 1 illustrates a practical case of PTT target with 6 realized sub-targets, their 240 and 18 CEM corresponding thermal dose surfaces and their MR T2 axial images performed intraoperatively and at $48 \mathrm{~h}$ after the procedure. In Figure 2, we show intra- and postoperative lesion measurements in 31 cases of PTT.

Mean time between first sonication and MR T2 axial images used for intraoperative measurement was $117 \pm 39.9 \mathrm{~min}$ and $42.7 \pm 4.7 \mathrm{~h}$ for the postoperative scan as shown in Figure 2A for 31 consecutive cases. Mean axial T2 lesion diameter was $6.6 \pm 1 \mathrm{~mm}$ immediately after the treatment and $9.0 \pm 1.0 \mathrm{~mm}$ postoperatively. Mean axial T2 measurements of the lesions $(n=$ 31) were $6.34 \mathrm{~mm}$ [SD: 1.0 , CI 99\% $(5.88 ; 6.80)]$ in mediolateral and $6.95 \mathrm{~mm}$ [SD: $0.9, \mathrm{CI} 99 \%(6.5 ; 7.3)]$ in the anteroposterior dimensions (black ellipse in Figures 2B,C). Forty eight hours after the procedure, they were $8.9 \mathrm{~mm}$ [SD:1.1, CI 99\% (8.4; 9.4)] and $9.1 \mathrm{~mm}$ [SD: 0.9, CI 99\% $(8.7,9.5)]$, respectively. Mean measured intraoperative 240 CEM thermal dose diameters in the mediolateral direction were 4.8 [SD:1.0, CI99\% (4.4; 5.3)] and $5.6 \mathrm{~mm}$ [SD: 0.9 , CI $99 \%(4.4 ; 5.3)$ ] anteroposteriorly (dark blue ellipse in Figures 2B,C). The 18 CEM thermal dose diameters were $7.5 \mathrm{~mm}$ [SD: 1.4, CI 99\% $(6.95 ; 8.16)]$ and $7.8 \mathrm{~mm}$ [SD: 1.0 , CI 99\% $(7.3 ; 8.3)$ ], respectively (light blue ellipse in Figures 2B,C).

Statistical analysis in 31 PTT targets displayed in Figure 3 revealed good correlations (1) between mean axial T2 lesion diameter intraoperatively and the mean 240 CEM thermal dose diameter ( $r=0.52$, Figure 3A), (2) between the mean axial T2 lesion diameter 48 hours post treatment and the mean $18 \mathrm{CEM}$ thermal dose diameter $(r=0.62$, Figure 3B), and (3) between the mean axial T2 lesion diameter intraoperatively and $48 \mathrm{~h}$ post-treatment ( $r=0.62$, Figure $3 \mathrm{C})$.

An example of insufficient target coverage despite repeated sonications in a patient treated before this study series is illustrated in Figure 4. In a second step before this series, longer sonication durations were applied. Spatial coverage was significantly increased as shown in Figure 5 but was nevertheless insufficient in some patients. Figure 6 illustrates how our current approach can be successfully 

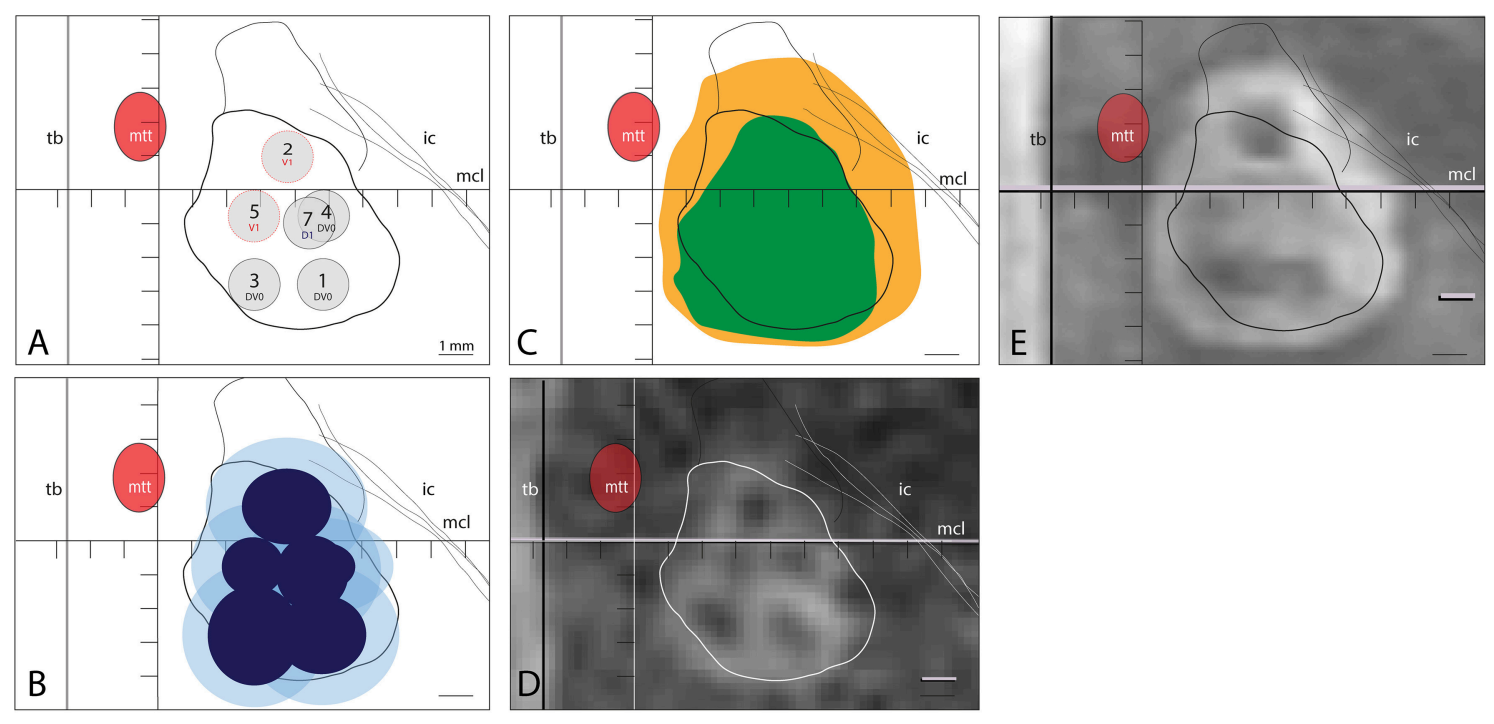

FIGURE 1 | (A) Applied target sub-units 1,3 and 4 at plan DV0, 2 and 5 at V1 and 7 at D1. (B) 240 CEM thermal dose surfaces reached (dark blue) in sub-target 1 $(2.6 \times 2.3 \mathrm{~mm}), 2(2.6 \times 2.2), 3(2.7 \times 2.9), 4(4.1 \times 2.8), 5(1.8 \times 1.7)$ and $7(2.1 \times 2.2) .18 \mathrm{CEM}$ thermal dose surfaces (light blue) reached in sub-target $1(4.7 \times 4)$, $2(4.5 \times 4.1), 3(4.2 \times 4.2), 4(4.1 \times 2.8), 5(3.6 \times 3.6), 7(1.8 \times 1.2$ and $4 \times 3.9)$. (C) shows the projection of the axial T2 MR lesions measured intraoperatively (green, corresponding to $\mathbf{D}$ ) and at $48 \mathrm{~h}$ post-treatment (orange, corresponding to $\mathbf{E}$ ), displayed at high magnification.
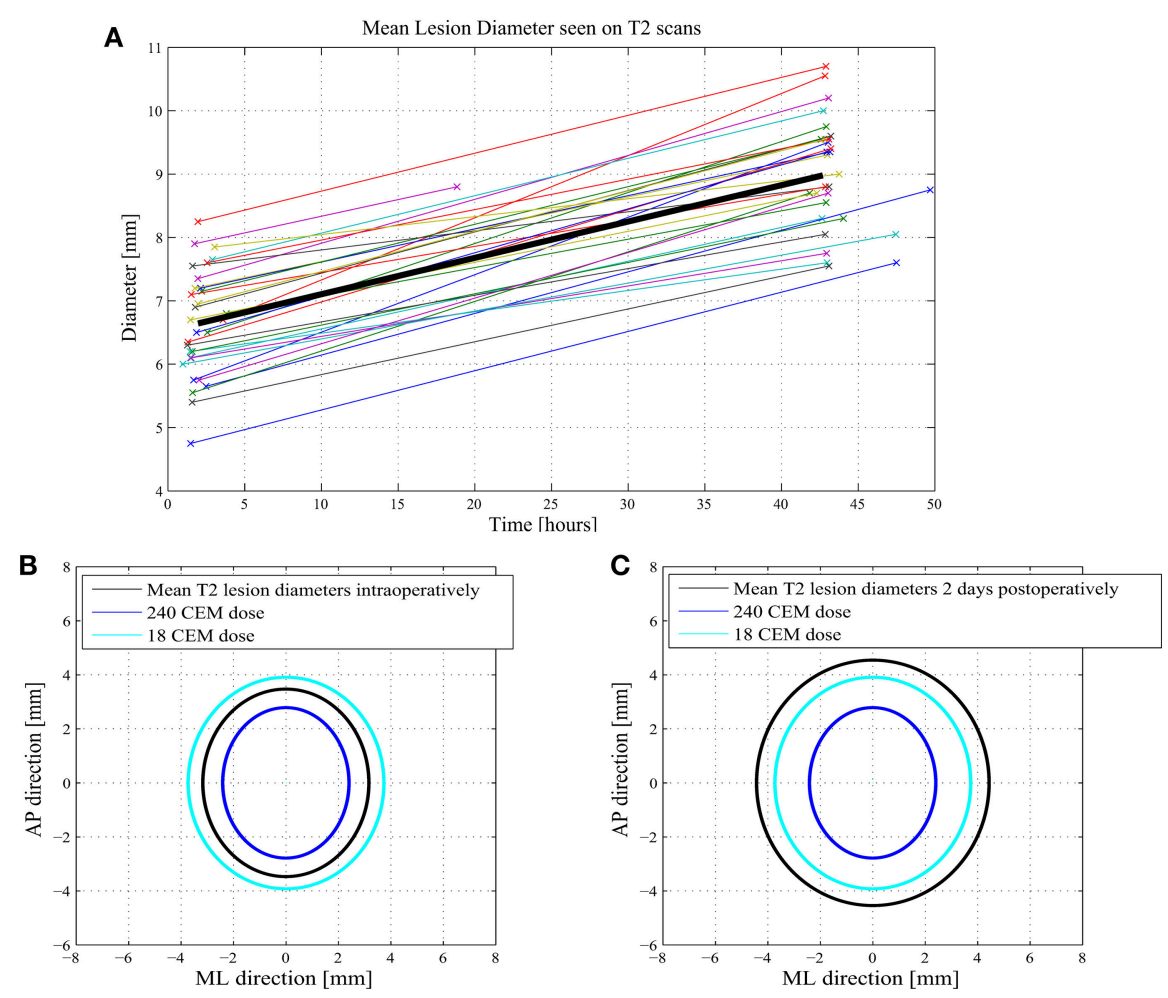

FIGURE 2 | (A) shows 31 cases with measurements of their mean lesion diameter in axial T2 intra- and postoperatively. (B) shows intraoperative mean axial T2 measurements of the lesions as well as their 240 and 18 CEM thermal dose mediolateral and anteroposterior diameters. (C) shows the postoperative T2 lesion diameters at 2 days with their thermal doses. 

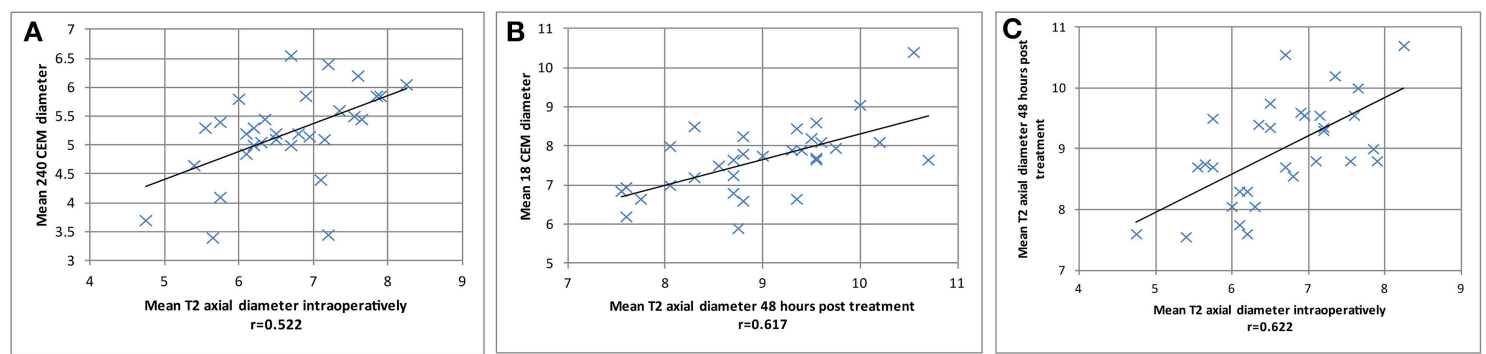

FIGURE 3 | Thirty one PTT targets are displayed for correlations between mean axial T2 lesion diameter intraoperatively and the mean 240 CEM thermal dose diameter in (A) $(r=0.52)$. In (B) mean T2 axial diameter $48 \mathrm{~h}$ post treatment was correlated with the mean $18 \mathrm{CEM}$ thermal dose diameter $(r=0.62)$. In $\mathbf{( C )}$, mean axial T2 lesion diameter intraoperatively was correlated with mean T2 axial diameter 48 h post-treatment $(r=0.62)$.
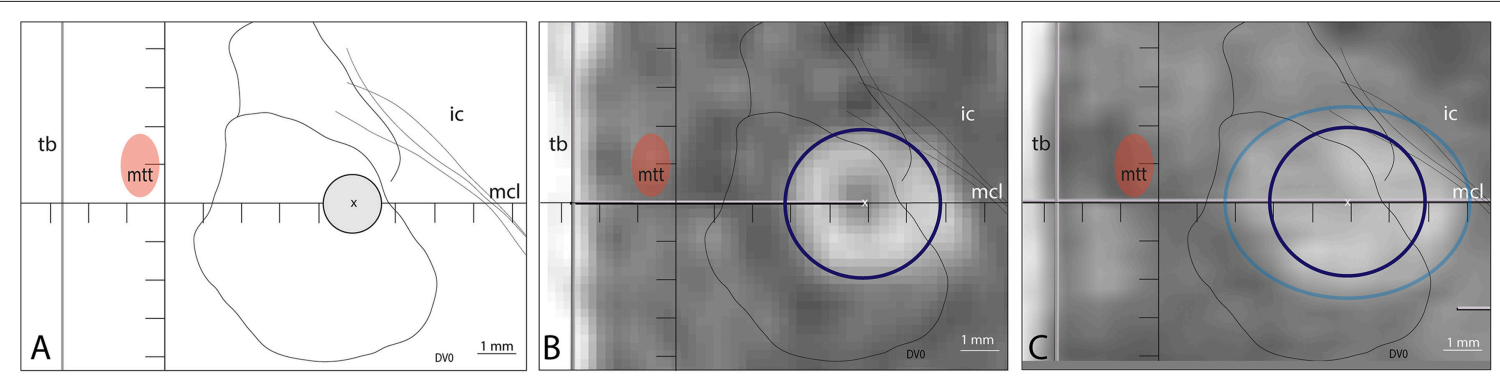

FIGURE 4 | Example of insufficient target coverage of a patient treated before this study series which led to initially good but later partial therapeutic effect on the symptoms. (A) Target was chosen at (L7.5, MCL, DVO) and repeated sonications $(n=4)$ with temperatures ranging from 55 to $57^{\circ} \mathrm{C}$ and of $13 \mathrm{~s}$ duration were applied on the same spot. The 240 CEM thermal dose outline (dark blue circle) was projected in (B) on the axial T2 scan intraoperatively $(4 \times 3.8 \mathrm{~mm}$ ) and both 18 CEM (light blue, $6.3 \times 4.9 \mathrm{~mm}$ ) and 240 CEM thermal dose outlines are shown in $(\mathbf{C})$ on the T2 axial scan performed 2 days after the treatment.
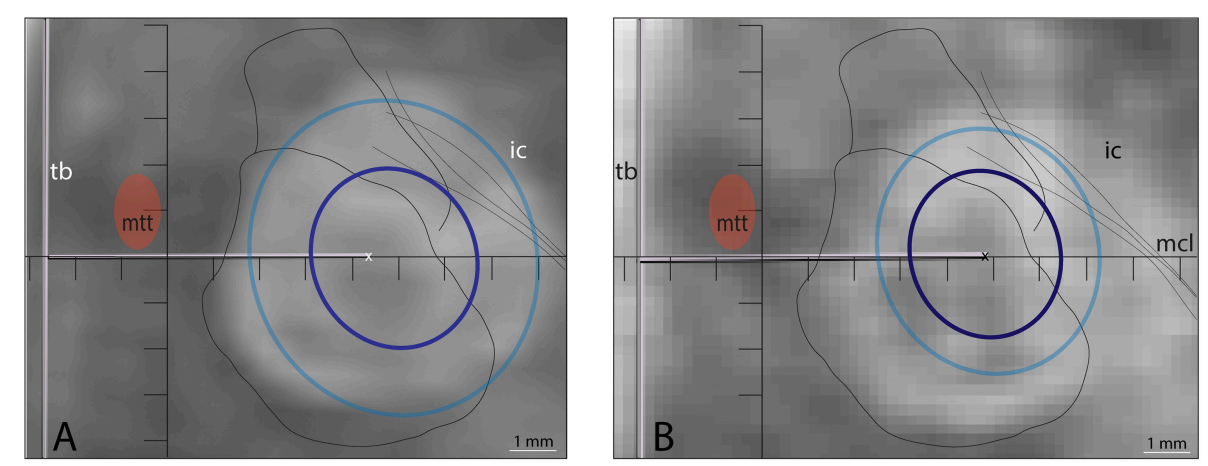

FIGURE 5 | Two patients in whom longer sonication durations were applied treated before this series. Spatial coverage was increased as shown in the thermal dose values for 240 CEM but as shown in the axial T2 scan $48 \mathrm{~h}$ after the treatment, the hyperintense signal was difficult to associate with any thermal dose equivalent. In (A) temperatures reached in final sonications were 56, 56, and $54^{\circ} \mathrm{C}$ (sonication time: 28, 28, and 20 s, power: 450, 500, and 600 W) with final $18 \mathrm{CEM}$ (light blue) and $240 \mathrm{CEM}$ (dark blue) surfaces in axial projections of $6.1 \times 6.8$ and $3.5 \times 3.9 \mathrm{~mm}$, respectively. In B: temperature reached was $57^{\circ} \mathrm{C}$ in $24 \mathrm{~s}(450 \mathrm{~W})$ for final 18 and 240 CEM thermal dose surfaces of $4.7 \times 5.4$ and $3.2 \times 3.6 \mathrm{~mm}$, respectively. In (A,B), the PTT single target coordinates were L7.5, MCL, and DVO.

applied to complement an insufficiently covered PTT target performed previously.

\section{DISCUSSION}

Targeted thresholds of 18 and 240 CEM are based on experimental data in the rabbit and primate brain summarized by MacDannold and co-workers $(3,4)$. The thermal dose threshold of 240 CEM is thought to represent the LD100, or $100 \%$ probability to achieve necrosis at the corresponding isothermal surface, and the $18 \mathrm{CEM}$ value represents a $50 \%$ probability for thermal damage. Imaging and histological correlations with a thermal dose based approach have been published in animals $(4,11-17)$ and in humans $(18,19)$. Little is known about tissue specific (white matter vs. gray matter) variations in the human brain as well as tissue changes with increasing 

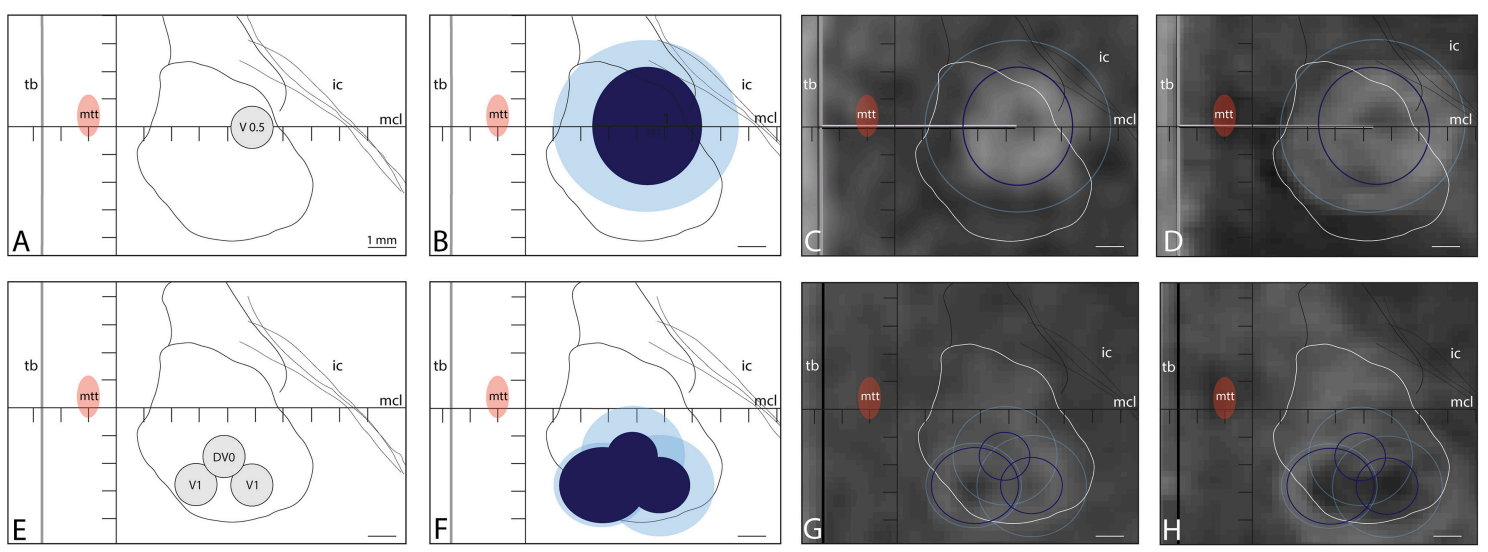

FIGURE 6 | Two treatments performed in the same patient within 2 years (A-D and later $\mathbf{E}-\mathbf{H})$ because of insufficient target coverage and partial symptom recurrence. (A,B) show the target placed at L7.5, MCL, and V0.5. Temperatures reached in final sonications were $55,56,58,57$, and $55^{\circ} \mathrm{C}$ (sonication time: $13 \mathrm{~s}$ for all, power: 350,450 , and $500 \mathrm{~W}$ ) with final $18 \mathrm{CEM}$ (light blue) and $240 \mathrm{CEM}$ (dark blue) thermal dose surfaces on axial projections of $6.6 \times 6.1 \mathrm{~mm}$ and $3.9 \times 4.2 \mathrm{~mm}$, respectively. (C,D) show the lesions in axial T2 scan performed intraoperatively (C) and 2 days after it (D) with projections of their 18 and 240 CEM thermal dose outlines. In a second session, a complement of PTT was planned (E) in 3 target sub-units: 1 (L7.5, MCL-2.7, V1), 2 (L5.5, MCL-2.7, V1), and 3 (L6.5, MCL-1.7, DV0). Temperatures reached in final sonications were 55,56 , and $55^{\circ} \mathrm{C}$ (sonication time: 10 , 10, and 16 s, power: 550,550 , and $650 \mathrm{~W}$ ). Final 18 and $240 \mathrm{CEM}$ thermal dose surfaces were shown in (F). (G,H) show the 240 and 18 CEM thermal dose outlines projected on axial T2 scans performed intraoperatively $(\mathbf{G})$ and 2 days after it $\mathbf{( H )}$.

age. Hints about different tissue thresholds can already be found in the older ultrasound literature $(20,21)$. The approach described here concerns a fiber tract target, in which a relevant part of heat diffusion may take place along its course. We expect more homogeneous perilesional heat diffusion patterns in nuclear targets (thalamus and pallidum). These observations warrant future detailed analyses of perilesional heat diffusion in nuclear targets.

Bringing together the correlative data of this study and histological animal data (4), the following can be proposed. Intraoperative T2 imaging zones I and II (10) correspond to the sum of the 240 CEM and a part, the most central, of the 18 CEM thermal dose area (Figure 2B), and thus to the 6 to $7 \mathrm{~mm}$ large thermolesioned tissue domain Figure 3A shows a good correlation between the intraoperative T2 imaging and the 240 CEM thermal dose area. The comparison between the T2 zone I and II surface areas intraoperatively and at 2 days allows to document the amount of edema arising during the first 2 postoperative days (Figures 2C, 3C). Figure 3B shows a good correlation between the T2 imaging of zone I and II area after 2 days and the 18 CEM thermal dose area. These areas are larger than the thermolesioned domain, and they correlate well with each other. This allows to foresee at the end of the intervention how large the zone I and II will be at 2 days, and to know that their extension is non-lesional and due to the development of intralesional cytotoxic and perilesional vasogenic edema. A mentioned above, it is only the most central part of the 18 CEM area which contains thermolesioned tissue. Concerning study limitations, histological data which have allowed to develop the thermal dose based lesioning parameters are all derived from animal experiments, and should be transposed with care to humans, as they surely cannot include for example the changes underwent by the aging human brain tissue. Secondly, there are no volume calculations because all measurements were performed only on axial planes, i.e., two-dimensionally. Thirdly, we still lack knowledge about the cumulative effect of thermal doses: there is rising but not yet quantified evidence that they can be summed up over one single target, thus allowing a successful thermal lesioning even in patients with unfavorable skull absorption preventing the application of a 240 CEM thermal dose in a single application.

\section{CONCLUSION}

This study presents our current approach to the MRgFUS pallidothalamic lesioning which was developed because the two strategies of application repetitions and increase of application duration failed to prevent symptom recurrences or partial symptom control. It takes into account interindividual variability, and is characterized by the applications of multiple small thermal lesions using shortest possible durations, and distributed in a preplanned manner to optimize target coverage. Using thermal dose monitoring rather than energy- or temperature-based approaches sticks with the biophysics of focused ultrasound based tissue thermal lesioning and provides a correlative and predictive dimension. This one is essential when considering sufficient lesion coverage of targets and safety for surrounding structures in MRgFUS functional neurosurgical interventions.

Our clinical results (in preparation) show as expected a clear-cut reduction of symptom recurrences or partial symptom control over the first postoperative year.

\section{ETHICS STATEMENT}

No ethical approval was sought because MRgFUS PTT is approved by the Swiss Health State Department and covered by 
swiss social insurances. All patients treated with this protocol signed an informed consent form after having been fully informed about the treatment, its results and risks.

\section{AUTHOR CONTRIBUTIONS}

MG, DJ, and DM: conception and design. MG and DM: acquisition of data. MG, DM, CF, and DJ: analysis and interpretation of data. All authors: drafting the article, critically revising the article, and reviewed submitted version of manuscript. MG: approved the final version of the manuscript

\section{REFERENCES}

1. Magara A, Bühler R, Moser D, Kowalski M, Pourtehrani P, Jeanmonod D. First experience with MR-guided focused ultrasound in the treatment of Parkinson's disease. J Ther Ultrasound. (2014) 2:11. doi: 10.1186/2050-5736-2-11

2. Gallay MN, Moser D, Federau C, Jeanmonod D. Anatomical and technical reappraisal of the pallidothalamic tractotomy with the incisionless transcranial MR-guided focused ultrasound. A technical note. Front Surg. (2019) 6:2. doi: 10.3389/fsurg.2019.00002

3. McDannold N, Vykhodtseva N, Jolesz FA, Hynynen K. MRI investigation of the threshold for thermally induced blood-brain barrier disruption and brain tissue damage in the rabbit brain. Magn Reson Med. (2004) 51:913-23. doi: $10.1002 / \mathrm{mrm} .20060$

4. McDannold N, Livingstone M, Top CB, Sutton J, Todd N, Vykhodtseva N. Preclinical evaluation of a low-frequency transcranial MRI-guided focused ultrasound system in a primate model. Phys Med Biol. (2016) 61:7664-87. doi: 10.1088/0031-9155/61/21/7664

5. Meshorer A, Prionas SD, Fajardo LF, Meyer JL, Hahn GM, Martinez AA. The effects of hyperthermia on normal mesenchymal tissues. Application of a histologic grading system. Arch Pathol Lab Med. (1983) 107:328-34.

6. Moser D, Zadicario E, Schiff G, Jeanmonod D. Measurement of targeting accuracy in focused ultrasound functional neurosurgery. Neurosurg Focus. (2012) 32:E2. doi: 10.3171/2011.10.FOCUS11246

7. Moser D, Zadicario E, Schiff G, Jeanmonod D. MR-guided focused ultrasound technique in functional neurosurgery: targeting accuracy. J Ther Ultrasound. (2013) 1:3. doi: 10.1186/2050-5736-1-3

8. Gallay MN, Moser D, Rossi F, Pourtehrani P, Magara AE, Kowalski M, et al. Incisionless transcranial MR-guided focused ultrasound in essential tremor: cerebellothalamic tractotomy. J Ther Ultrasound. (2016) 4:5. doi: 10.1186/s40349-016-0049-8

9. Gallay MN, Moser D, Jeanmonod D. Safety and accuracy of incisionless transcranial MR-guided focused ultrasound functional neurosurgery: singlecenter experience with 253 targets in 180 treatments. J Neurosurg. (2018) 5:1-10. doi: 10.3171/2017.12.jns172054

10. Martin E, Jeanmonod D, Morel A, Zadicario E, Werner B. High-intensity focused ultrasound for noninvasive functional neurosurgery. Ann Neurol. (2009) 66:858-61. doi: 10.1002/ana.21801

11. Elias WJ, Khaled M, Hilliard JD, Aubry J-F, Frysinger RC, Sheehan JP, et al. A magnetic resonance imaging, histological, and dose modeling comparison of focused ultrasound, radiofrequency, and Gamma Knife radiosurgery lesions in swine thalamus. J Neurosurg. (2013) 119:307-17. doi: $10.3171 / 2013.5$.JNS122327

12. Hynynen K, Vykhodtseva NI, Chung AH, Sorrentino V, Colucci V, Jolesz FA. Thermal effects of focused ultrasound on the brain: determination with MR imaging. Radiology. (1997) 204:247-53. doi: 10.1148/radiology.204.1.9205255

13. Hynynen K, McDannold N, Clement G, Jolesz FA, Zadicario E, Killiany R, et al. Pre-clinical testing of a phased array ultrasound system for MRI-guided on behalf of all authors. MG and CF: statistical analysis. DM: administrative/technical/material support.

\section{FUNDING}

CF is supported by the Swiss National Science Foundation.

\section{ACKNOWLEDGMENTS}

We thank Dr. Payam Pourtherani, Dr. Mike Fitze, and Dr. Oskar Blosser at Rodiag Diagnostic Centers Solothurn for MR imaging.

noninvasive surgery of the brain-a primate study. Eur J Radiol. (2006) 59:14956. doi: 10.1016/j.ejrad.2006.04.007

14. Marquet F, Boch A-L, Pernot M, Montaldo G, Seilhean D, Fink M, et al. Noninvasive ultrasonic surgery of the brain in non-human primates. J Acoust Soc Am. (2013) 134:1632-9. doi: 10.1121/1.4812888

15. McDannold N, Moss M, Killiany R, Rosene DL, King RL, Jolesz FA, et al. MRIguided focused ultrasound surgery in the brain: tests in a primate model. Magn Reson Med. (2003) 49:1188-91. doi: 10.1002/mrm.10453

16. Vykhodtseva N, Sorrentino V, Jolesz FA, Bronson RT, Hynynen K. MRI detection of the thermal effects of focused ultrasound on the brain. Ultrasound Med Biol. (2000) 26:871-80. doi: 10.1016/S0301-5629(00)00216-7

17. Vykhodtseva NI, Hynynen K, Damianou C. Histologic effects of high intensity pulsed ultrasound exposure with subharmonic emission in rabbit brain in vivo. Ultrasound Med Biol. (1995) 21:969-79.

18. Bond AE, Shah BB, Huss DS, Dallapiazza RF, Warren A, Harrison $M B$, et al. Safety and efficacy of focused ultrasound thalamotomy for patients with medication-refractory, tremor-dominant parkinson disease: a randomized clinical trial. JAMA Neurol. (2017) 74:1412-8. doi: 10.1001/jamaneurol.2017.3098

19. Federau C, Goubran M, Rosenberg J, Henderson J, Halpern CH, Santini $\mathrm{V}$, et al. Transcranial MRI-guided high-intensity focused ultrasound for treatment of essential tremor: a pilot study on the correlation between lesion size, lesion location, thermal dose, and clinical outcome. J Magn Reson Imaging. (2018) 48:58-65. doi: 10.1002/jmri.25878

20. Basauri L, Lele PP. A simple method for production of trackless focal lesions with focused ultrasound: statistical evaluation of the effects of irradiation on the central nervous system of the cat. J Physiol. (1962) 160:513-34.

21. Meyers R, Fry WJ, Fry FJ, Dreyer LL, Schultz DF, Noyes RF. Early experiences with ultrasonic irradiation of the pallidofugal and nigral complexes in hyperkinetic and hypertonic disorders. J Neurosurg. (1959) 16:32-54. doi: $10.3171 /$ jns.1959.16.1.0032

Conflict of Interest Statement: MG, DM, and DJ were employed by company SoniModul Ltd, Center of Ultrasound Functional Neurosurgery, Solothurn, Switzerland.

The remaining author declares that the research was conducted in the absence of any commercial or financial relationships that could be construed as a potential conflict of interest.

Copyright (c) 2019 Gallay, Moser, Federau and Jeanmonod. This is an open-access article distributed under the terms of the Creative Commons Attribution License (CC $B Y)$. The use, distribution or reproduction in other forums is permitted, provided the original author(s) and the copyright owner(s) are credited and that the original publication in this journal is cited, in accordance with accepted academic practice. No use, distribution or reproduction is permitted which does not comply with these terms. 